\title{
Utilização de Dispositivos Móveis por Idosos de um Programa de Extensão Universitária
}

\author{
Sandra Maria Mendes Rodrigues Pereira - UERJ, UNIRIO, smrpereira19@gmail.com \\ Maria Cardoso de Castro Berry - UERJ, dramariaberry@gmail.com \\ Maria Fátima G. Menezes - UERJ, m.fatima.menezes@gmail.com \\ Maria Isabel de Castro de Souza - UERJ, profamariaisabel@yahoo.com.br
}

\section{Resumo}

O objetivo desse estudo foi identificar o uso de dispositivos móveis em idosos de um programa de extensão universitária. Trata-se de um estudo quantitativo descritivo de uma amostra de 50 idosos entrevistados presencialmente no "Programa Interdisciplinar de Saúde e Qualidade de Vida do Idoso". Os resultados mostraram que $86 \%$ utilizam celular; $60 \%$ usam aplicativos móveis, sendo que destes, todos utilizam o WhatsApp; 56,7\% utilizam rede social tipo Facebook e apenas um idoso já baixou um aplicativo sobre Alimentação ou Saúde. Percebe-se assim uma maior utilização de aplicativos voltados para comunicação. Esse estudo mostrou que a maioria dos idosos entrevistados tem celulares com recursos, porém, não os utilizam na amplitude de atividades que poderiam ser realizadas.

Palavras-chave: aprendizagem móvel, aplicativos móveis, dispositivos móveis, educação em saúde, idoso.

\begin{abstract}
The aim of this study was to identify the use of mobile devices in the elderly people of an university extension program. This is a descriptive quantitative study of a sample of 50 elderly interviewed in person from the "Interdisciplinary Health and Elderly Quality of Life Program". The results showed that $86 \%$ use the mobile phone; $60 \%$ use mobile applications, and of these, all use WhatsApp; 56,7\% use Facebook-type social network and only an elderly already downloaded an app about Food or Health. It is noticed a greater use of apps aimed on communication. This study showed that most of the elderly interviewed have mobile phones with resources, but don't use them in the range of activities that could be used.
\end{abstract}

Elderly use of Mobile Devices in an University Extension Program

Keywords: mobile learning, mobile applications, mobile devices, health education, elderly.

\section{Introdução}

O processo de envelhecimento populacional ocorre em quase todo o mundo devido à queda inicial das taxas de mortalidade, posterior redução nas taxas de natalidade e extensão da expectativa de vida, trazendo desafios distintos em vários setores da sociedade, como na economia, educação e saúde. Sendo assim, é necessário desenvolver estratégias para garantir um envelhecimento ativo e com qualidade de vida a todos os cidadãos e a educação é fundamental nesse processo. No Brasil existem vários programas de extensão universitária voltados para o público de idosos, com modelos pedagógicos diversos, que têm contribuído para o protagonismo dessa faixa etária (Alcântara et al., 2016). O fator idade não pode ser considerado impedimento para que um indivíduo aprenda, tendo em vista a constante renovação e modificações neuronais que acontecem ao longo de sua vida (Souza 
e Aragon, 2018). Ações educativas são estratégias de promoção de saúde e contribuem na manutenção do papel social do idoso trazendo, além do conhecimento, um resgate da autoestima e autonomia, tornando o sujeito ativo e participativo. Além disso, a inserção da maturidade na educação, possibilita uma melhor compreensão das transformações do mundo, favorecendo a interação entre as gerações (Garcia et al., 2019).

As tecnologias digitais têm criado novas formas de aprendizagem e divulgação de conhecimentos na atualidade. Os aparelhos celulares, que antes serviam apenas para enviar e receber ligações e/ou mensagens, ganharam outras utilidades e serviços, como os aplicativos (apps) desenvolvidos especialmente para estes aparelhos. Eles são extremamente versáteis, com diversas finalidades (entretenimento, educação, saúde, bem-estar, transporte e etc.) e podem ser baixados em plataformas diferentes (De Oliveira e de Menezes Alencar, 2017). Os aplicativos móveis podem colaborar na melhoria de qualidade de vida e autonomia de idosos, porém, alguns trazem crenças infundadas, algumas vezes reforçadas pelos próprios familiares, em relação a dificuldades na aprendizagem de assuntos relacionados às tecnologias de informação e comunicação (TICs) e quanto antes eles se inserirem no contexto das tecnologias, melhores serão seus sentimentos de inclusão e interação na sociedade (Bragagnolo e Deon, 2017). No Brasil, ocorre uma concentração na proporção de internautas na faixa etária compreendida entre 10 e 34 anos $(63,5 \%)$, enquanto que a faixa etária a partir de 60 anos representa apenas 5,3\% da amostra, reforçando a desigualdade entre as gerações (TIC Domicílios 2017).

Apesar da insegurança de alguns idosos, o envelhecimento populacional estimulou o mercado de diversos aplicativos voltados para esse público, dentre eles, os da área de saúde, são recursos importantes, pois podem influenciar o estilo de vida, promover o envelhecimento ativo e saudável e melhorar a autonomia dos idosos no cuidado da sua própria saúde, além disso, despertam o interesse e a curiosidade, contribuindo também para a sua inclusão digital. Porém, apesar dos benefícios apontados, esses recursos tecnológicos carecem de maiores estudos e investigações, sendo necessário o desenvolvimento de interfaces que atendam às necessidades específicas dos idosos (Amorim et al., 2018). Sugere-se adaptar a interface às suas características, considerando o tamanho e o tipo de fonte, o tamanho dos ícones, o contraste nas cores, assim como o design de interação, que deve ser mais intuitivo (Carvalho et al., 2016). Um material educativo digital voltado para idosos deve oferecer segurança, ser atrativo, promover participação e interação e incluir temas que contribuam para a melhoria da qualidade de vida, criando novas formas de ensino e aprendizagem (Barbosa et al., 2017).

A educação para a saúde representa um processo pedagógico centrado na autonomia do indivíduo, estimulando mudanças a partir de sua capacidade crítica. $\mathrm{O}$ uso de aplicativos de saúde pode auxiliar no desenvolvimento e na disseminação de informações de forma lúdica e, ao mesmo tempo, séria, motivando a escolha de hábitos saudáveis (Da Rocha et al., 2017). O envelhecimento contínuo da população afeta a área da saúde, pois, à medida que envelhece, a população passa a apresentar um perfil epidemiológico diferenciado, caracterizado pelo aumento progressivo da prevalência de doenças crônicas não transmissíveis, como diabetes e doença arterial coronariana, entre outras, do qual decorre a crescente demanda por cuidados de longa duração (Brasil 2018). Manter a autonomia e independência de idosos é a meta primordial tanto para os próprios indivíduos que envelhecem quanto para os setores de planejamento na área de saúde (Saad, 2016).

Nesse sentido, a rede de atenção ao idoso pode ser intensificada com o uso de aplicativos desenvolvidos por profissionais especializados, com informações atualizadas e fidedignas repercutindo positivamente não apenas na saúde e qualidade de vida dessa população, mas também no processo educacional, ampliando seus conhecimentos e desenvolvendo práticas que estimulem sua inclusão digital. 
O presente estudo teve como objetivo identificar o uso de dispositivos móveis em uma amostra de idosos que participam de um programa de extensão universitária.

\section{Metodologia}

O estudo foi desenvolvido no Hospital Universitário Gaffreé e Guinlhe (HUGG) da Universidade Federal do Estado do Rio de Janeiro (UNIRIO), local de aplicação de questionário sobre a utilização de dispositivos móveis por uma amostra de idosos.

O método utilizado nesse estudo foi de pesquisa quantitativa descritiva com a aplicação de um questionário, com 10 perguntas objetivas, sendo duas relacionadas a informações pessoais do participante (idade e sexo) e oito sobre a utilização de dispositivos eletrônicos (três sobre quais dispositivos utilizam, uma sobre o sistema operacional, três sobre quais funções e aplicativos são utilizados no celular e uma sobre o grau de dificuldade da utilização desses aplicativos). A fim de conhecer possíveis dificuldades de compreensão do questionário, foi aplicado um teste piloto com 5 idosos. Observou-se que alguns não identificavam o WhatsApp como um aplicativo e no questionário final então, este foi exemplificado como aplicativo de comunicação). O questionário foi aplicado presencialmente, através de entrevista, pelo pesquisador responsável e por alunos devidamente treinados, entre novembro de 2018 e janeiro de 2019, em uma amostra de conveniência de 50 idosos que participam do programa de extensão universitária "Programa Interdisciplinar de Saúde e Qualidade de Vida do Idoso", grupo Renascer, da Universidade Federal do Estado do Rio de Janeiro (UNIRIO). O questionário faz parte do projeto de pesquisa: "Alterações no estado nutricional de idosos acompanhados em um hospital universitário do Rio de Janeiro" e foi submetido e aprovado pelo Comitê de Ética em Pesquisa da UNIRIO e registrado sob o número CAAE 63109616.9.0000.5258. Os aspectos éticos foram respeitados, considerando a Resolução 196/96 do Ministério da Saúde, através do Termo de Consentimento Livre e Esclarecido.

Após a coleta de dados os resultados foram tabulados e analisados de forma descritiva.

\section{Resultados e Discussão}

A idade dos participantes variou de 61 a 89 anos, sendo a mediana 74 anos de idade. Dos 50 componentes da amostra, a maioria, 92\%, era do sexo feminino $(n=46)$. Essa maior participação do gênero feminino também foi encontrada em outras amostras de idosos que participam de programas de extensão universitária (Mota et al., 2018, Oliveira et al., 2018).

Em relação ao uso de dispositivos eletrônicos, $12 \%$ dos idosos $(n=6)$ não utilizam nenhum dispositivo, $30 \%(n=15)$ relataram usar o computador, sendo que $2 \%(n=1)$ relataram usar computador e tablet, $2 \%(\mathrm{n}=1)$ computador, tablet e celular e $26 \%(\mathrm{n}=13)$ computador e celular, ou seja, nenhum relatou usar apenas o computador; $6 \%(\mathrm{n}=3)$ usam tablet ou iPad e $86 \%$ ( $\mathrm{n}=43$ ) usam celular, sendo que 58\% (n=29) utilizam apenas este dispositivo. (Gráfico 1). 


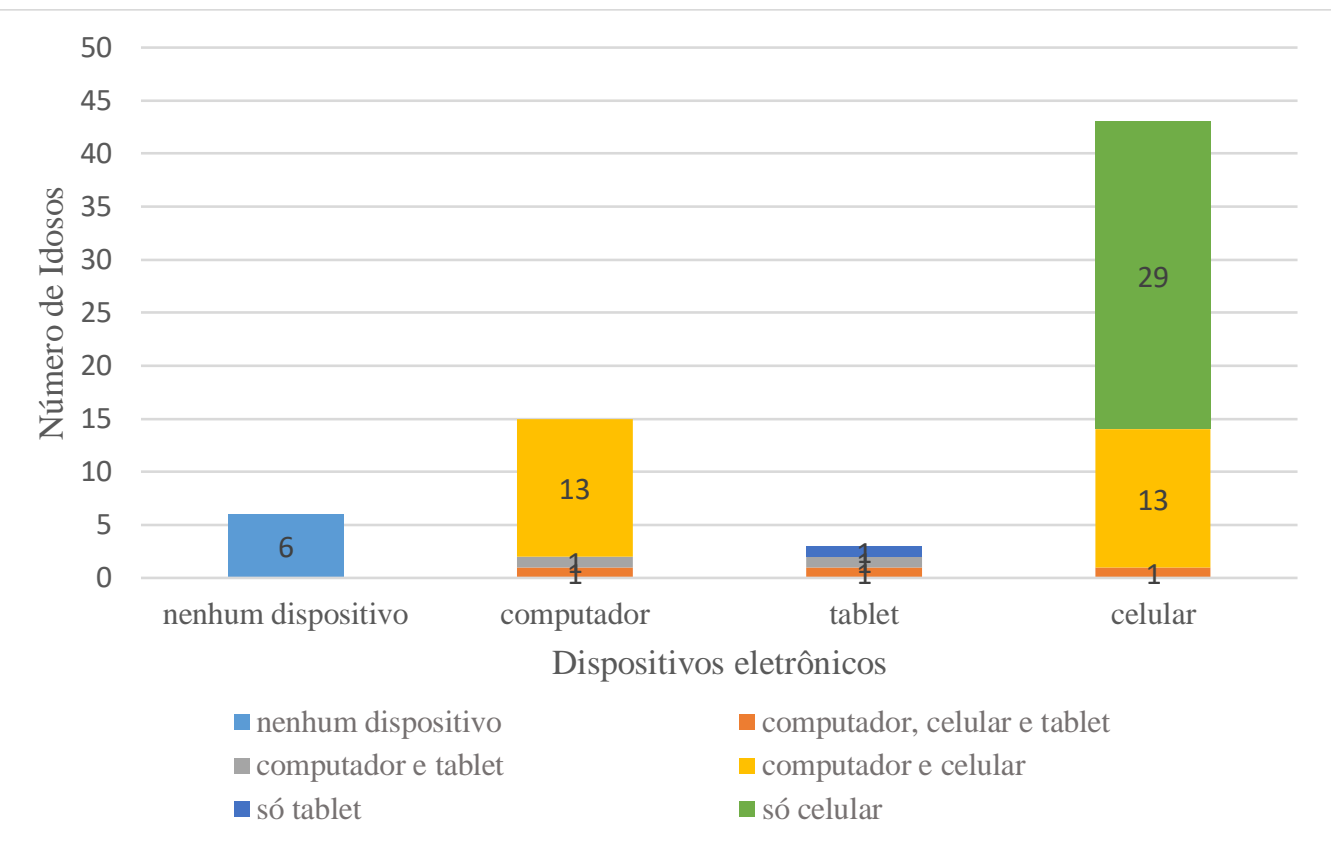

Gráfico 1 - Uso de dispositivos eletrônicos pelos idosos entrevistados do grupo Renascer

A maior utilização do celular como dispositivo eletrônico está de acordo com dados do Comitê Gestor da Internet no Brasil (CGI.br 2018) que indicam que 83\% da população brasileira possui telefone celular. Deve-se ressaltar a importância deste dispositivo como promotor da inclusão digital, principalmente pela sua mobilidade. Diagnóstico feito pela Estratégia Brasileira para a Transformação Digital (E-Digital 2018) constatou que o acesso móvel à internet já é maior do que por meio de computadores: em 2014, 76\% dos usuários de internet acessavam por meio do celular, contra $80 \%$ que utilizavam o computador. Em 2016, o uso do celular para internet subiu para 94\%, enquanto o acesso por computador caiu para 49\%. Além da mobilidade, os celulares apresentam outra vantagem, Rocha et al. (2016) constataram em um grupo de idosos que a interface Touchscreen facilitou a execução de tarefas pois concentra a atenção apenas na tela, não sendo necessário o foco simultâneo em 3 pontos: teclado, mouse e tela.

Quanto a utilização do celular, dos 43 idosos que possuem este dispositivo, todos o utilizam para "realizar/ receber ligações"; $69,7 \%(\mathrm{n}=30)$ relataram utilizar o aparelho para "enviar/ receber mensagens WhatsApp"; 65,1\% (n=28) "fotografar e filmar", 60,5\% (n=26) "receber e enviar mensagens de texto (SMS) " e 39,5\% (n=17) entrar em redes sociais tipo Facebook. (Gráfico 2). 


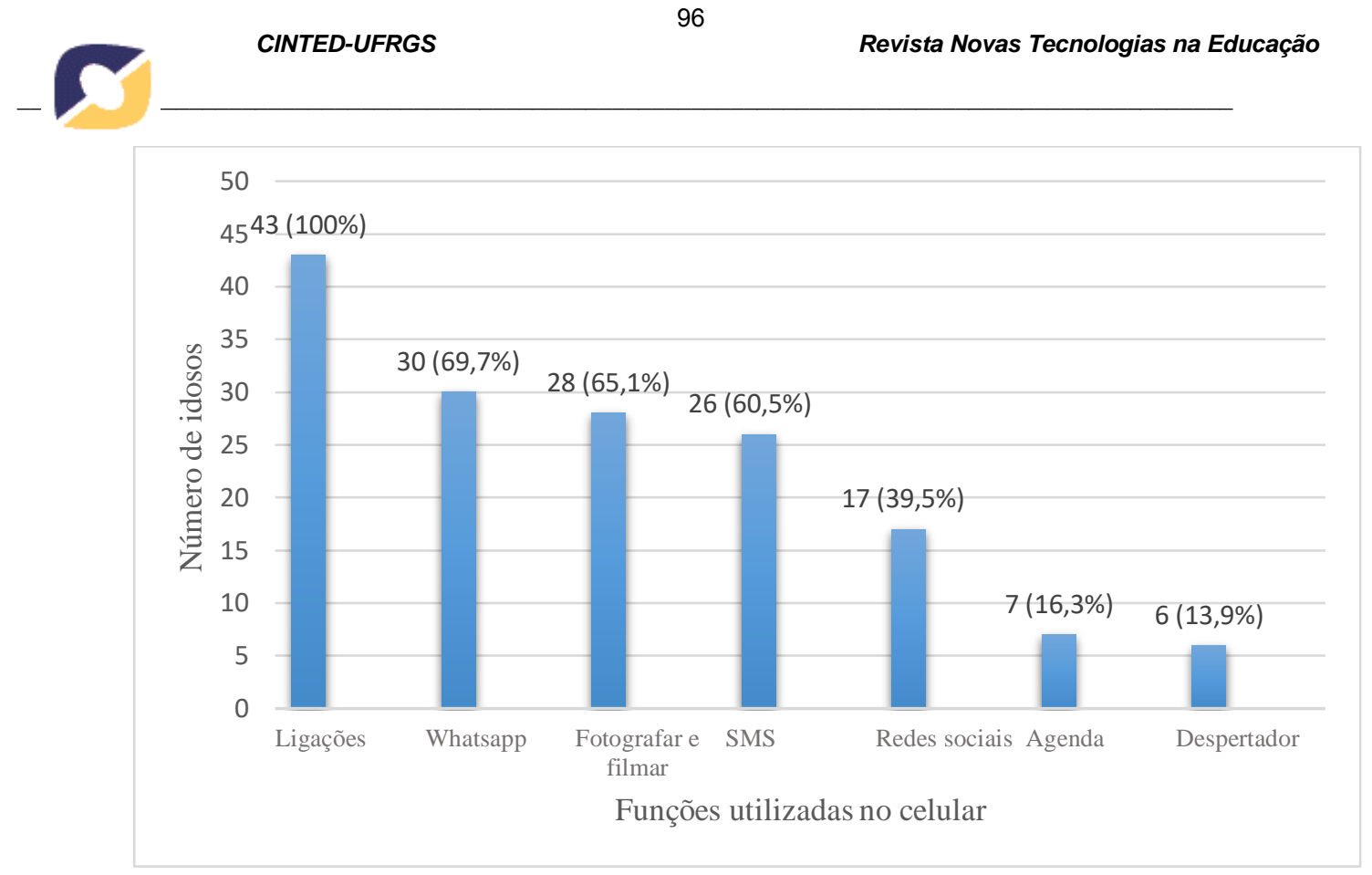

Gráfico 2 - Funções utilizadas no celular pelos idosos entrevistados do grupo Renascer

Segundo dados da TIC Domicílios 2017, em relação às atividades realizadas no celular pelos idosos, 95\% relataram "fazer e receber" chamadas telefônicas, 37\% utilizaram para fotos, 34\% enviaram mensagens via WhatsApp e $18 \%$ SMS, $18 \%$ utilizaram o celular para entrar em redes sociais tipo Facebook e 8\% acessaram jogos. Comparando esses dados com os desse estudo, percebe-se que apenas o percentual da função "fazer e receber ligações" está em consonância, os idosos dessa amostra utilizam mais as outras funções: WhatsApp, fotografar, SMS e redes sociais tipo Facebook, demonstrando interesse nas áreas de comunicação e registro de atividades.

Dos idosos que relataram usar celular, 33 têm acesso à internet e a maioria destes, $88 \%(\mathrm{n}=29)$ possuem o sistema operacional Android, 3\% ( $\mathrm{n}=1)$ iOS, 3\% $(\mathrm{n}=1)$ windows e 6\% (n=2) não sabiam. (Gráfico 3).

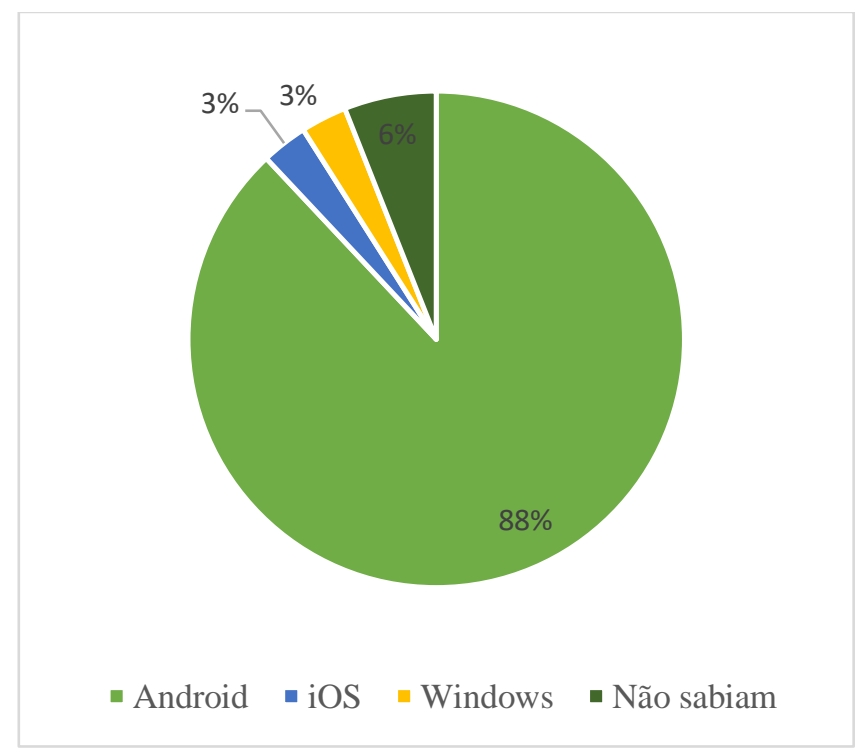

Gráfico 3 - Sistema operacional dos celulares utilizados pelos idosos entrevistados do grupo Renascer 
Em relação ao uso de aplicativos no celular, $60 \%(\mathrm{n}=30)$ dos idosos relatou usar algum, sendo que todos utilizam o WhatsApp. Além deste, os aplicativos mais relatados foram: rede social tipo Facebook 56,7\% ( $\mathrm{n}=17)$, aplicativo de transporte privado $36,7 \%(\mathrm{n}=11)$, notícias $33,3 \%(\mathrm{n}=10)$, clima $26,7 \%(n=8)$ e culinária $23,3 \%(n=7)$; apenas $3,3 \%(n=1)$ já baixou um aplicativo sobre Alimentação ou Saúde. A participação em redes sociais tipo Facebook pode estar associada ao fato do programa de extensão do qual esses idosos participam ter uma página no Facebook onde as atividades realizadas são divulgadas. Essas práticas são importantes para as interações sociais e redução do isolamento. (Gráfico 4).

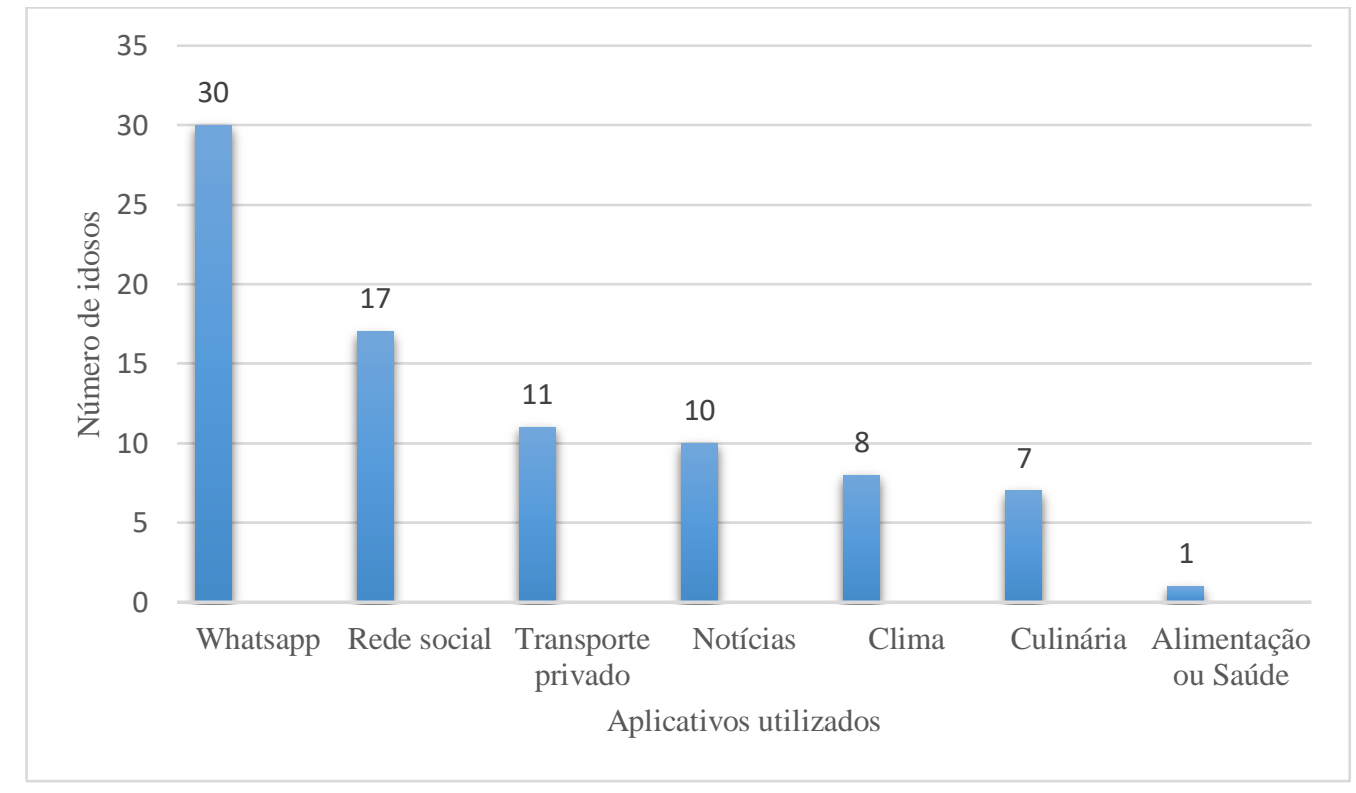

Gráfico 4 - Aplicativos utilizados pelos idosos entrevistados do grupo Renascer

Percebe-se uma maior utilização de aplicativos voltados para comunicação e busca de informações gerais. Esses dados estão em consonância com os da TIC Domicílios 2017, que relataram também, uma baixa utilização por parte dos idosos de aplicativos relacionados à saúde. Talvez essa pouca utilização se deva a pequena oferta desses aplicativos direcionados especificamente para idosos. Amorim et al. (2018) fizeram uma busca em smartphones com sistemas operacionais Android e $i O S$ utilizando os descritores 'idoso', 'saúde do idoso', 'envelhecimento', 'elderly' e 'salud de los ancianos'. Consideraram aplicativos voltados para a saúde do idoso aqueles que abordassem a promoção da saúde física e cognitiva e/ou a prevenção de condições e de eventos nocivos à saúde do idoso, como quedas, fragilidade e dependência funcional. Excluindo-se os aplicativos voltados para a vida social do idoso, direcionados para uma doença específica ou aqueles voltados ao diagnóstico e a avaliação em saúde e os direcionados para familiares ou cuidadores, os resultados mostraram apenas 5 aplicativos disponíveis na língua portuguesa: 1 para estimulação de práticas de exercícios físicos, 1 para prevenção de quedas, 1 para estimulação cognitiva e 2 para busca de profissionais de saúde. Percebe-se assim, a pouca oferta desses recursos com informações gerais sobre saúde, que possam ser utilizadas na rotina dos idosos.

Quando questionados sobre a facilidade na utilização de aplicativos, dos 30 idosos que os utilizam, 66,7\% $(n=20)$ consideraram fácil, 16,65\% $(n=5)$ nem fácil nem difícil e 16,65\% $(n=5)$ consideraram difícil. $\mathrm{O}$ fato da maioria não apresentar dificuldades, sugere que a prática reduz $\mathrm{o}$ distanciamento do idoso com ambientes digitais. (Gráfico 5). 

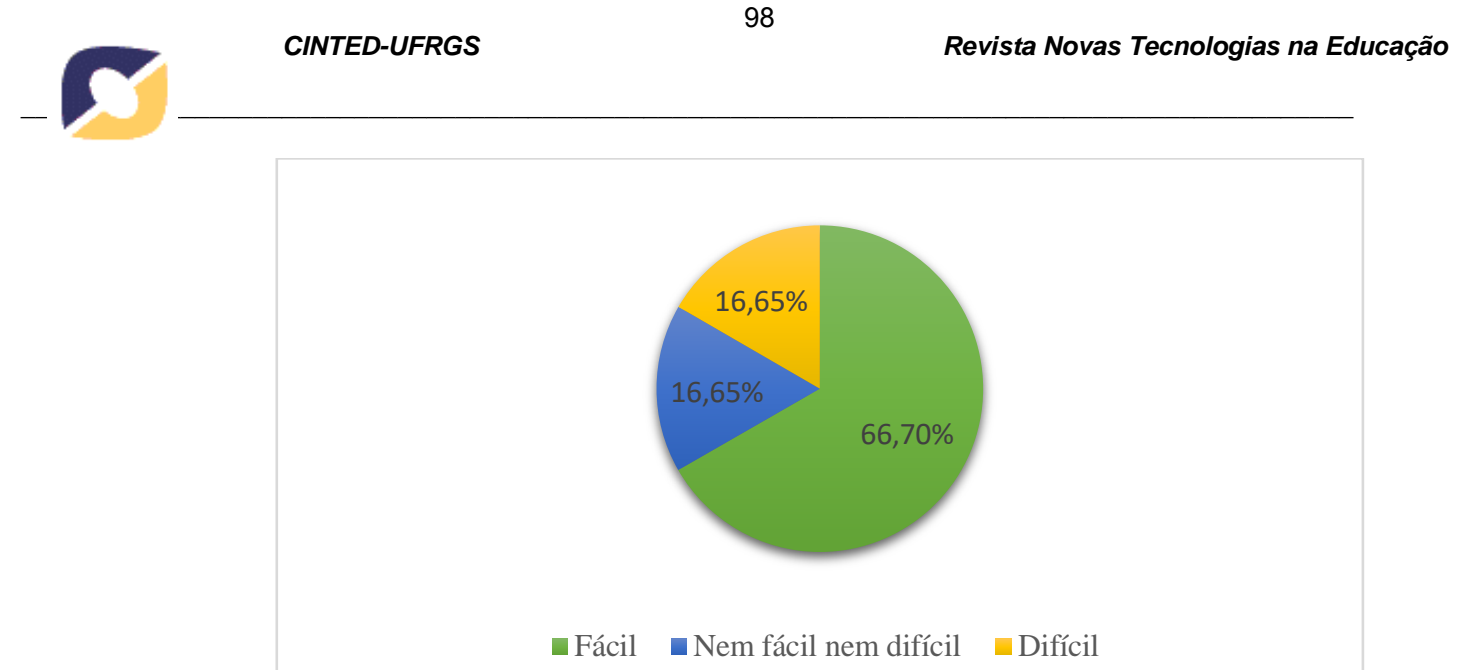

Gráfico 5 - Facilidade na utilização de aplicativos pelos idosos entrevistados do grupo Renascer

Apesar de não terem sido questionados sobre a não utilização de aplicativos no celular, alguns fizeram questão de se justificar: "tenho medo de errar e quebrar o aparelho"; "quero aprender"; "fico chateada pois meus netos e filhos não têm paciência para me explicar"; "tenho vontade de aprender mas tenho pouca memória"; "não enxergo". Esses sentimentos de medo de danificar equipamentos ou travar sistemas também foram relatados por Dos Santos e Almêda (2017) ao analisarem o uso das TICs por idosos de um programa de iniciativa do governo federal realizado na cidade de Florânia/RN, porém, eles também expressaram sentimentos de felicidade e encantamento por estarem aprendendo e interagindo com algo novo, reforçando que o processo de inclusão digital é de extrema relevância para o idoso, já que o estimula a superar o medo e vislumbrar novas possibilidades de aprendizagem. Alvim et al. (2017) também relatam que, apesar de certa resistência, os idosos reconhecem a necessidade de, por algum meio, adentrar no mundo digital e buscam se atualizar por meio de cursos, oficinas e palestras de inclusão, alfabetização e letramento digitais. Batista et al. (2019) num estudo de relato de experiência de um projeto de inclusão digital de ação extensionista, em um núcleo interdisciplinar de cuidados e pesquisa a adultos e idosos em condição crônica, observaram que antes do curso, as alunas eram inseguras, com pouca ou nenhuma habilidade com computadores e periféricos e com o desenvolvimento do curso houve evolução não apenas de conhecimentos adquiridos, mas também na parte motora, psíquica, social e analítica de cada participante. Rompendo o medo da tecnologia, as participantes evoluíram muito no processo ensino-aprendizado.

\section{Conclusão}

A utilização das tecnologias de informação e comunicação (computador, tablet, celulares, smartphones e outros dispositivos digitais conectados à $W E B$ ), vem modificando a vida cotidiana das pessoas em geral.

Percebe-se nesse estudo que a maioria dos idosos entrevistados tem celulares com recursos e apesar de alguns relatarem insegurança, eles demonstraram interesse em utilizá-los. Os dados mostraram uma maior utilização de aplicativos na área da comunicação, que naturalmente os torna mais próximos de seus amigos e familiares, reduzindo o isolamento social. Apesar deste ponto positivo observa-se que esta faixa etária poderia ser mais beneficiada se ampliasse seu uso através de atividades nos dispositivos móveis que estimulem outras formas de aprendizagem. Além dos equipamentos que oferecem serviços e recursos digitais, é preciso incentivo e adaptação desses recursos para o público da terceira idade. Esses resultados reforçam a necessidade de projetos de inclusão digital para idosos que desmistifiquem e exercitem a utilização de novas tecnologias, contribuindo para uma melhor convivência de idosos na sociedade contemporânea. 
Sendo assim, as universidades podem representar um exponencial agente modificador dessa realidade, não só promovendo ações educativas para idosos como cursos de atualização em informática, mas também no desenvolvimento de tecnologias adaptadas a esse público, principalmente aplicativos móveis, já que os dispositivos móveis são os mais utilizados. Deve-se estimular principalmente os voltados para a promoção da saúde, pois, como visto, muitos idosos são portadores de doenças crônicas e informações técnicas atualizadas inseridas num contexto inovador, podem contribuir na adesão de escolhas mais saudáveis, melhorando sua qualidade de vida e sua autonomia além de contribuir para sua inclusão digital.

\section{Referências Bibliográficas}

AlCÂntARA, A. de O.; CAMARANO, A. A.; GIACOMIN, K. C. (org.). Política Nacional do Idoso: velhas e novas questões. 2016. Disponível em:

http://www.ipea.gov.br/portal/index.php?option=com_content\&view=article\&id=28693. Acesso em: 10 jul. 2019.

ALVIM, K. C. B. L.; DE SOUSA ROCHA, F.; CHARIGLIONE, I. P. F. S. O idoso e o uso da tecnologia-uma revisão sistemática da literatura. Revista Kairós: Gerontologia, v. 20, n. 4, p. 295313, 2017. Disponível em:

https://www.researchgate.net/publication/331208004_O_idoso_e_o_uso_da_tecnologia_uma_revisa o_sistematica_da_literatura_REVISTA_KAIROS_GERONTOLOGIA_v_20_p_295-313_2017.

Acesso em: 10 jun. 2019.

AMORIM, D. N. P.; SAMPAIO, L. V. P.; CARVALHO, G. A.; VILAÇA, K. H. C. Aplicativos móveis para a saúde e o cuidado de idosos. Revista Eletrônica de Comunicação, Informação e Inovação em Saúde, v. 12, n. 1, 2018. Disponível em: https://www.reciis.icict.fiocruz.br/index.php/reciis/article/view/1365. Acesso em: 10 jun. 2019.

BARBOSA, M. L. K.; HAHN, C. S. R.; SAMPAIO, D. C. F.; ROSA, L. Q.; CAZELLA, S. C.; MACHADO, L. R. Envelhecer@ Saudável: um material educacional digital voltado ao público idoso. RENOTE, v. 15, n. 1, 2017. Disponível em: https://seer.ufrgs.br/renote/article/view/75098. Acesso em: 10 jun. 2019.

BATISTA, E. B.; SILVA, L. W. S.; MOURA, L. R.; QUEIROZ, V. A. R. de; MATOS, R. S.; SILVA, S. J. L.; SANTOS, G. S.; LIMA, I. S. S.; NERY, N. T. R.; RODRIGUES, A. A. Inclusão Digital como ferramenta ao envelhecimento ativo: um relato de experiência. Prisma.com, n. 38, p. 69-81, 2019. Disponível em: http://ojs.letras.up.pt/index.php/prismacom/article/view/5428/5151. Acesso em: 05 jul. 2019.

BRAGAGNOLO, S. M.; DEON, M. R. Inclusão Digital para a Terceira Idade. Revista Visão: Gestão Organizacional, v. 6, n. 2, 2017. Disponível em: https://periodicos.uniarp.edu.br/index.php/visao/article/view/1232. Acesso em: 07 jan. 2019.

BRASIL, Ministério da Saúde. Secretaria de Atenção à Saúde Departamento de Ações Programáticas e Estratégicas. Orientações técnicas para a implementação de Linha de Cuidado para Atenção Integral à Saúde da Pessoa Idosa no Sistema Único de Saúde - SUS [recurso eletrônico] / Ministério da Saúde, Secretaria de Atenção à Saúde, Departamento de Ações Programáticas e Estratégicas. - Brasília: Ministério da Saúde, 2018. Disponível em: http://bvsms.saude.gov.br/bvs/publicacoes/linha_cuidado_atencao_pessoa_idosa.pdf. Acesso em: 10 jul. 2019. 
CARVALHO, E.; ARANTES, R. C.; CINTRA, A. S. R. The inclusion of elderly persons from the Instituto Henrique da Silva Semente (IHESS) in Indaiatuba, São Paulo, in the digital age: physiogerontological contributions. Revista Brasileira de Geriatria e Gerontologia. Rio de Janeiro, v. 19, n.4, p. 567-575, Aug. 2016. Disponível em: http://www.scielo.br/scielo.php?script=sci_arttext\&pid=S180998232016000400567\&lng=en\&nrm=iso. Acesso em: 08 set. 2019.

CGI.br/NIC.br, Centro Regional de Estudos para o Desenvolvimento da Sociedade da Informação (Cetic.br), Pesquisa sobre o Uso das Tecnologias de Informação e Comunicação nos domicílios brasileiros - TIC Domicílios 2018. Disponível em:

http://data.cetic.br/cetic/explore?idPesquisa=TIC_DOM\&idUnidadeAnalise $=$ Usuarios \&ano=2018 Acesso em: 21 set. 2019.

DE OLIVEIRA, A. R. F.; DE MENEZES ALENCAR, M. S. O uso de aplicativos de saúde para dispositivos móveis como fontes de informação e educação em saúde. RDBCI: Revista Digital de Biblioteconomia e Ciência da Informação, v. 15, n. 1, p. 234-245, 2017. Disponível em: https://periodicos.sbu.unicamp.br/ojs/index.php/rdbci/article/view/8648137. Acesso em: 07 jan. 2019.

DOS SANTOS, R. F.; ALMÊDA, K. A. O Envelhecimento Humano e a Inclusão Digital: análise do uso das ferramentas tecnológicas pelos idosos. Ciência da Informação em Revista, v. 4, n. 2, p. 59-68, 2017. Disponível em: http://www.seer.ufal.br/index.php/cir/article/view/3146. Acesso em: 07 jan. 2019.

E-DIGITAL: Estratégia Brasileira para a Transformação Digital - E-Digital. BRASIL 2018. Disponível em: http://www.mctic.gov.br/mctic/export/sites/institucional/estrategiadigital.pdf. Acesso em: 10 ago. 2019.

GARCIA, K. R.; CHIARELlO, M. D.; LIMA, J. T. P. S.; PEREIRA, L. C.; FUNGHETTO, S. S.; KARNIKOWSHI, M. G. O. Inserção de pessoas na maturidade na educação superior. Revista de Divulgação Científica Sena Aires, v. 8, n. 2, p. 132-138, 2019. Disponível em: http://revistafacesa.senaaires.com.br/index.php/revisa/article/view/391. Acesso em: 10 ago. 2019.

MOTA, R. L. R.; SOUZA, N. R.; ROSSI, V. H. C.; SANTOS, P. G. M.; GUIMARÃES, R. E. Educação em Saúde para Idosos em uma Universidade Aberta para Maturidade. Revista Eixos Tech, v. 5, n. 1, 2018. Disponível em: http://eixostech.pas.ifsuldeminas.edu.br/ojs/index.php/eixostech/article/view/158. Acesso em: 10 ago. 2019.

OLIVEIRA, L. L. M.; OLIVEIRA, B. P.; VIANNA, S. O.; LANA, D. C. P. Perfil clínico e sócio demográfico dos idosos participantes de um programa de universidade aberta ao idoso. Sinapse Múltipla, v. 7, n. 2, p. 206-210, 2018. Disponível em: http://periodicos.pucminas.br/index.php/sinapsemultipla/article/view/18886. Acesso em: 15 mar. 2019.

ROCHA, F. S.; SANTANA, E. B.; SILVA, E. S.; CARVALHO, J. S. M.; CARVALHO, F. L. Q. Uso de apps para a promoção dos cuidados à saúde. In: SEMINÁRIO DE TECNOLOGIAS APLICADAS EM EDUCAÇÃO E SAÚDE, 3. 2017, Bahia. Anais eletrônicos [...]. [Salvador?]: UNEB, 2017. Disponível em: https://www. revistas.uneb.br/index.php/staes/article/download/3832/2382. Acesso em: 15 mar. 2019.

ROCHA, R. G. O.; FARIA, J. R. G.; SALES, A. B.; BRITO, R.; AMARAL, M. A.; SALES, M. B. Inclusão Digital de Pessoas Idosas: Um Estudo de Caso utilizando Computadores Desktop e 


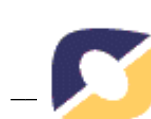

Tablets. RENOTE, v. 14, n. 1, 2016. Disponível em: https://seer.ufrgs.br/renote/article/view/67329. Acesso em: 10 ago. 2019.

SAAD, P. M. Envelhecimento populacional: demandas e possibilidades na área de saúde. Séries Demográficas, v. 3, p. 153-166, 2016. Disponível em:

http://www.abep.org.br/publicacoes/index.php/series/article/view/71. Acesso em: 07 jan. 2019.

SOUZA, S. S. dos S. de; ARAGON, G. T. Estilos de Aprendizagem e Ensino a Distância na Perspectiva da Inclusão. EAD EM FOCO, [S.1.], v. 8, n. 1, jun. 2018. ISSN 2177-8310. Disponível em: http://eademfoco.cecierj.edu.br/index.php/Revista/article/view/668/327. Acesso em: 28 ago. 2019. doi:https://doi.org/10.18264/eadf.v8i1.668.

TIC DOMICÍLIOS 2017 Pesquisa Sobre o Uso das Tecnologias de Informação e Comunicação nos Domicílios Brasileiros. Comitê Gestor da Internet no Brasil. Disponível em:

https://www.cetic.br/media/docs/publicacoes/2/tic_dom_2017_livro_eletronico.pdf. Acesso em: 20 jul. 2019. 